\title{
Bring Back 'Permanent' Filters
}

\author{
Leo P. Lawler ${ }^{1}$
}

Received: 16 May 2016/Accepted: 19 May 2016/Published online: 1 June 2016

(C) Springer Science+Business Media New York and the Cardiovascular and Interventional Radiological Society of Europe (CIRSE) 2016

To the Editor,

It is time to remove 'temporary' and 'retrievable' descriptors from the vernacular of IVC filter placement. Neither practitioner nor company could ever claim complete control over the many factors that determine retrievability. Such terminology misleads referrers and patients alike. By raising unrealistic expectations, it also unnecessarily gifts a novel caseload to an already adversarial medico-legal system. Although an innovation of good intention, these redundant discriminators have been hijacked through marketing, misinformation, and misinterpretation to the detriment of this common vital practice.

Patients should only be offered a 'permanent' filter albeit with a design that may permit, but never guarantee, a method of removal. The moniker 'permanent' filter better articulates the risk that it may well be and better focuses all minds on the threshold for justified use based on clinical indications and patient reservations. It is better for all to offer a permanent filter that is potentially retrievable than vice versa.

Clear communication is a basic tenet of patient care, so for the good of all, let us make this simple language change in our practice and our literature and re-educate the world on filters.

\section{Compliance with Ethical Standards}

Conflict of interest No conflict of interest.

Research involving animal and human rights This article does not contain any studies with human participants or animals performed by any of the authors.

Informed consent Informed consent does not apply.

Leo P. Lawler

llawler@mater.ie

1 Mater Misericordiae University Hospital, Eccles Street, Dublin 7, Ireland 